\title{
ON GENERALIZATIONS OF $p$-SETS AND THEIR APPLICATIONS
}

\author{
HENG ZHOU AND ZHIQIANG XU
}

\begin{abstract}
The $p$-set, which is in a simple analytic form, is well distributed in unit cubes. The well-known Weil's exponential sum theorem presents an upper bound of the exponential sum over the $p$-set. Based on the result, one shows that the $p$-set performs well in numerical integration, in compressed sensing as well as in UQ. However, $p$-set is somewhat rigid since the cardinality of the $p$-set is a prime $p$ and the set only depends on the prime number $p$. The purpose of this paper is to present generalizations of $p$-sets, say $\mathcal{P}_{d, p}^{\mathbf{a}, \epsilon}$, which is more flexible. Particularly, when a prime number $p$ is given, we have many different choices of the new $p$-sets. Under the assumption that Goldbach conjecture holds, for any even number $m$, we present a point set, say $\mathcal{L}_{p, q}$, with cardinality $m-1$ by combining two different new $p$-sets, which overcomes a major bottleneck of the $p$-set. We also present the upper bounds of the exponential sums over $\mathcal{P}_{d, p}^{\mathbf{a}, \epsilon}$ and $\mathcal{L}_{p, q}$, which imply these sets have many potential applications.
\end{abstract}

Key words and phrases $p$-set; Deterministic sampling; Numerical integral; Exponential sum

AMS Subject Classification 2000 11K38, 65C05, 11L05, 41A10, 65D32

\section{INTRODUCTION}

1.1. $p$-set. Let $p$ be a prime number. We consider the point set

$$
\mathcal{P}_{d, p}=\left\{\mathbf{x}_{0}, \ldots, \mathbf{x}_{p-1}\right\} \subset[0,1)^{d}
$$

where

$$
\mathbf{x}_{j}=\left(\left\{\frac{j}{p}\right\},\left\{\frac{j^{2}}{p}\right\}, \ldots,\left\{\frac{j^{d}}{p}\right\}\right) \in[0,1)^{d}, \quad j \in \mathbb{Z}_{p},
$$

$\mathbb{Z}_{p}:=\{0,1, \ldots, p-1\}$ and $\{x\}$ is the fractional part of $x$ for a nonnegative real number $x$. The point set $\mathcal{P}_{d, p}$ is called $p$-set and was introduced by Korobov [5] and Hua-Wang 4. Recently, $p$-set attracts much attention since its advantage in numerical integration [1, in the recovery of sparse trigonometric polynomials [12] and in the UQ [13. In [1, Dick presents a numerical integration formula based on $\mathcal{P}_{d, p}$ with showing the error bound of the formula depends only polynomially on the dimension $d$. In [12], Xu uses $\mathcal{P}_{d, p}$ to construct the deterministic sampling points of sparse trigonometric polynomials and show the sampling

Heng Zhou was supported by the National Natural Science Foundation of China (No. 61602341); Zhiqiang Xu was supported by NSFC grant $(11422113,91630203,11331012)$ and by National Basic Research Program of China (973 Program 2015CB856000). 
matrix corresponding to $\mathcal{P}_{d, p}$ has the almost optimal coherence. And hence, $\mathcal{P}_{d, p}$ has a good performance for the recovery of sparse trigonometric polynomials.

1.2. Extensions of $p$-set: $\mathcal{P}_{d, p}^{\mathbf{a}, \epsilon}$ and $\mathcal{L}_{p, q}$. The $p$-set is in a simple analytic form and hence it is easy to be generated by computer. However, the $p$-set is somewhat rigid with the point set only depending on a prime number $p$. If the function values at some points in $p$-set are not easy to be obtained, one has to change the prime number $p$ to obtain a new point set which has the different cardinality with the previous one. Hence, in practical application, it will be better that one has many different choices. We next introduce a generalization of $p$-set.

Let

$$
\mathbb{Z}_{p}^{d}:=\left\{\mathbf{a}=\left(a_{1}, \ldots, a_{d}\right) \in \mathbb{Z}^{d}: a_{j} \in \mathbb{Z}_{p}, j=1, \ldots, d\right\} .
$$

Suppose that $\mathbf{a}=\left(a_{1}, \ldots, a_{d}\right) \in \mathbb{Z}_{p}^{d}$ and $\epsilon=\left(\epsilon_{1}, \ldots, \epsilon_{d-1}\right) \in\{0,1\}^{d-1}$. We set

$$
\mathcal{P}_{d, p}^{\mathbf{a}, \epsilon}:=\left\{\mathbf{x}_{j}^{\mathbf{a}, \epsilon}: j \in \mathbb{Z}_{p}\right\}
$$

where

$$
\mathbf{x}_{j}^{\mathbf{a}, \epsilon}:=\left(\left\{\frac{a_{1} j}{p}\right\},\left\{\frac{a_{1}^{\prime} j+a_{2} j^{2}}{p}\right\}, \ldots,\left\{\frac{\sum_{h=1}^{d-1} a_{h}^{\prime} j^{h}+a_{d} j^{d}}{p}\right\}\right) \in[0,1)^{d}
$$

and $a_{k}^{\prime}=\epsilon_{k} a_{k}, k=1, \ldots, d-1$. We call $\mathcal{P}_{d, p}^{\mathbf{a}, \epsilon}$ as the $p$-set associating with the parameter a and $\epsilon$. If we take $\mathbf{a}=(1, \ldots, 1)$ and $\epsilon=(0, \ldots, 0)$, then $\mathcal{P}_{d, p}^{\mathbf{a}, \epsilon}$ is reduced to the classical $p$-set.

The $p$-set $\mathcal{P}_{d, p}^{\mathbf{a}, \epsilon}$ associating with the parameters $\mathbf{a}, \epsilon$ is more flexible. Given the prime number $p$, one can generate various point sets by changing the parameters a and $\epsilon$ with presenting an option set when the cardinality $p$ is given.

Note that the cardinality of both $\mathcal{P}_{d, p}^{\mathbf{a}, \epsilon}$ and $\mathcal{P}_{d, p}$ is prime. Since the distance between adjacent prime can be very large, the cardinality of $p$-set does not change smoothly. Using the set $\mathcal{P}_{d, p}^{\mathbf{a}, \epsilon}$, we next present a set with the cardinality being odd number. Suppose that $m \in 2 \mathbb{Z}$ is given. The Goldbach conjecture, which is one of the best-known unsolved problem in number theory, says that $m$ can be written as the sum of two primes, i.e., $m=p+q$ where $p$ and $q$ are prime numbers. One has verified the conjecture up to $m \leq 4 \cdot 10^{14}$ which is enough for practical application. We next suppose that $m=p+q$ with $p$ and $q$ being prime numbers. We set

$$
\mathcal{L}_{p, q}:=\left\{\begin{array}{cc}
\mathcal{P}_{d, p} \cup \mathcal{P}_{d, q}, & p \neq q \\
\mathcal{P}_{d, p}^{\mathbf{a}, \epsilon^{\prime}} \cup \mathcal{P}_{d, p}^{\mathbf{b}, \epsilon^{\prime \prime}}, & p=q,
\end{array}\right.
$$

where $\mathcal{P}_{d, p}^{\mathbf{a}, \epsilon^{\prime}}$ and $\mathcal{P}_{d, p}^{\mathbf{b}, \epsilon^{\prime \prime}}$ are the $p$-sets that we have defined above and $\mathbf{a}, \mathbf{b} \in \mathbb{Z}_{p}^{d}, \epsilon^{\prime}, \epsilon^{\prime \prime} \in$ $\{0,1\}^{d-1}$. We call $\mathcal{L}_{p, q}$ the $(p, q)$-set. As shown later, $\mathcal{P}_{d, p} \cap \mathcal{P}_{d, q}=\{(0, \ldots, 0)\}$ provided $p \neq q$. We can choose $\mathbf{a}, \mathbf{b}, \epsilon^{\prime}$ and $\epsilon^{\prime \prime}$ so that $\mathcal{P}_{d, p}^{\mathbf{a}, \epsilon^{\prime}} \cap \mathcal{P}_{d, p}^{\mathbf{b}, \epsilon^{\prime \prime}}=\{(0, \ldots, 0)\}$. Hence, under the assumption of Goldbach conjecture, for any odd number, says $m-1$, there exist $p, q$ so that $\left|\mathcal{L}_{p, q}\right|=p+q-1=m-1$.

We would like to mention the following point sets with cardinality $p^{2}$ [5, 4] :

$$
\begin{aligned}
& \mathcal{Q}_{p^{2}, d}=\left\{\mathbf{z}_{j}: j=0, \ldots, p^{2}-1\right\}, \mathbf{z}_{j}=\left(\left\{\frac{j}{p^{2}}\right\},\left\{\frac{j^{2}}{p^{2}}\right\}, \ldots,\left\{\frac{j^{d}}{p^{2}}\right\}\right) \in[0,1)^{d} \\
& \mathcal{R}_{p^{2}, d}=\left\{\mathbf{z}_{j, k}: j, k=0, \ldots, p-1\right\}, \mathbf{z}_{j, k}=\left(\left\{\frac{k}{p}\right\},\left\{\frac{j k}{p}\right\}, \ldots,\left\{\frac{j^{d-1} k}{p}\right\}\right) \in[0,1)^{d} .
\end{aligned}
$$


The weighted star discrepancy of $\mathcal{Q}_{p^{2}, d}$ and $\mathcal{R}_{p^{2}, d}$ is given in [2]. Using a similar method with above, we can generalize $\mathcal{Q}_{p^{2}, d}$ and $\mathcal{R}_{p^{2}, d}$ to $\mathcal{Q}_{p^{2}, d}^{\mathbf{a}, \epsilon}$ and $\mathcal{R}_{p^{2}, d}^{\mathbf{a}, \epsilon}$, respectively. We will introduce it in Section 2.3 in detail.

1.3. Organization. In Section 2, we present the upper bounds of the exponential sums over $\mathcal{P}_{d, p}^{\mathbf{a}, \epsilon}$ and $\mathcal{L}_{p, q}$. Particularly, we present the condition under which $\left|\mathcal{L}_{p, q}\right|=p+q-1$ and also prove that $\mathcal{P}_{d, p}^{\mathbf{a}, \epsilon} \cap \mathcal{P}_{d, p}^{\mathbf{b}, \epsilon}=\{(0, \ldots, 0)\}$ when $\mathcal{P}_{d, p}^{\mathbf{a}, \epsilon} \neq \mathcal{P}_{d, p}^{\mathbf{b}, \epsilon}$. We furthermore consider the generalization of the point sets $\mathcal{Q}_{p^{2}, d}$ and $\mathcal{R}_{p^{2}, d}$ and present the upper bounds of exponential sums over the new sets. The results in Section 2 show that the point sets presented in this paper have many potential applications in various areas. In Section 3 , we choose $\mathcal{L}_{p, q}$ as a deterministic sampling set for the recovery of sparse trigonometric polynomials and then show their performance.

\section{The exponential sums over $\mathcal{P}_{d, p}^{\mathbf{a}, \epsilon}$ AND $\mathcal{L}_{p, q}$}

The aim of this section is to present the exponential sums over $\mathcal{P}_{d, p}^{\mathbf{a}, \epsilon}$ and $\mathcal{L}_{p, q}$. To this end, we first introduce the well-known Weil's formula, which plays a key role in our proof.

Theorem 2.1. 11] Suppose that $p$ is a prime number. Suppose $f(x)=\sum_{h=1}^{d} m_{h} x^{h}$ with $m_{h} \in \mathbb{Z}(h=1, \ldots, d)$ and there is a $j \in\{1,2, \ldots d\}$, satisfying $p \nmid m_{j}$. Then

$$
\left|\sum_{x=1}^{p} e^{\frac{2 \pi \mathbf{i} f(x)}{p}}\right| \leq(d-1) \sqrt{p} .
$$

2.1. The exponential sum over $\mathcal{P}_{d, p}^{\mathbf{a}, \epsilon}$. Recall that

$$
\mathcal{P}_{d, p}^{\mathbf{a}, \epsilon}:=\left\{\mathbf{x}_{j}^{\mathbf{a}, \epsilon}: j \in \mathbb{Z}_{p}\right\}
$$

and

$$
\mathbf{x}_{j}^{\mathbf{a}, \epsilon}=\left(\left\{\frac{a_{1} j}{p}\right\},\left\{\frac{a_{1}^{\prime} j+a_{2} j^{2}}{p}\right\}, \ldots,\left\{\frac{\sum_{h=1}^{d-1} a_{h}^{\prime} j^{h}+a_{d} j^{d}}{p}\right\}\right) \in[0,1)^{d}
$$

where $\mathbf{a}=\left(a_{1}, \ldots, a_{d}\right) \in[1, p-1]^{d} \cap \mathbb{Z}^{d}, a_{j}^{\prime}=\epsilon_{j} a_{j}$ and $\epsilon=\left(\epsilon_{1}, \ldots, \epsilon_{d-1}\right) \in\{0,1\}^{d-1}$. Note that $\left|\mathcal{P}_{d, p}^{\mathbf{a}, \epsilon}\right|=p$. We next show the exponential sum formula over $\mathcal{P}_{d, p}^{\mathbf{a}, \epsilon}$.

Theorem 2.2. For any $\mathbf{k} \in[-p+1, p-1]^{d} \cap \mathbb{Z}^{d}$ and $\mathbf{k} \neq 0$, we have

$$
\left|\sum_{\mathbf{x} \in \mathcal{P}_{d, p}^{\mathbf{a}, \epsilon}} \exp (2 \pi \mathbf{i k} \cdot \mathbf{x})\right|=\left|\sum_{j=0}^{p-1} \exp \left(2 \pi \mathbf{i} \mathbf{k} \cdot \mathbf{x}_{j}^{\mathbf{a}, \epsilon}\right)\right| \leq(d-1) \sqrt{p} .
$$

Proof. Set

$$
g(j)=\sum_{\ell=1}^{d} c_{\ell} j^{\ell}
$$

where $c_{\ell}=k_{\ell} a_{\ell}+k_{\ell+1} a_{\ell}^{\prime}+\cdots+k_{d} a_{\ell}^{\prime}$. We set $j_{0}:=\max \left\{\ell: k_{\ell} \neq 0\right\}$. Then $c_{j_{0}}=k_{j_{0}} a_{j_{0}}$ and we have $p \nmid c_{j_{0}}$. According to Theorem [2.1, we obtain that

$$
\left|\sum_{j=0}^{p-1} \exp \left(2 \pi \mathbf{i k} \cdot \mathbf{x}_{j}^{\mathbf{a}, \epsilon}\right)\right|=\left|\sum_{j=0}^{p-1} \exp \left(2 \pi \mathbf{i} \frac{g(j)}{p}\right)\right| \leq(d-1) \sqrt{p}
$$


2.2. The exponential sum over $\mathcal{L}_{p, q}$. To this end, we consider the cardinality of $\mathcal{L}_{p, q}$. A simple observation is that $\left|\mathcal{L}_{p, q}\right| \leq p+q-1$. We would like to present the condition under which $\left|\mathcal{L}_{p, q}\right|=p+q-1$. We first consider the case where $p \neq q$.

Theorem 2.3. Suppose that $p$ and $q$ are two distinct prime numbers. Then $\left|\mathcal{L}_{p, q}\right|=p+q-1$.

Proof. According to (2), to this end, we just need show that

$$
\mathcal{P}_{d, p} \cap \mathcal{P}_{d, q}=\{(0, \ldots, 0)\} .
$$

We prove it by contradiction. Assume that $\mathcal{P}_{d, p} \cap \mathcal{P}_{d, q} \neq\{(0, \ldots, 0)\}$, and then there exists $j \in \mathbb{Z}_{p}^{*}:=\mathbb{Z}_{p} \backslash\{0\}$ and $k \in \mathbb{Z}_{q}^{*}$ so that $\left\{\frac{j^{i}}{p}\right\}=\left\{\frac{k^{i}}{q}\right\}, i=1, \ldots, d$. Particularly, we have $\frac{j}{p}=\frac{k}{q}$, which is equivalent to $j q=k p$. Since $p$ and $q$ are different prime numbers, $j \in \mathbb{Z}_{p}^{*}$ and $k \in \mathbb{Z}_{q}^{*}$, we have $j \mid k$ and $k \mid j$, which means $j=k$ and hence $p=q$. A contradiction.

We next consider the case where $p=q$, i.e., $\mathcal{L}_{p, q}=\mathcal{P}_{d, p}^{\mathbf{a}, \epsilon^{\prime}} \cup \mathcal{P}_{d, p}^{\mathbf{b}, \epsilon^{\prime \prime}}$. For the case where $\epsilon^{\prime}=\epsilon^{\prime \prime}$, we have

Theorem 2.4. Suppose that $\epsilon \in\{0,1\}^{d-1}$ is a fixed vector and $\mathbf{a}=\left(a_{1}, \ldots, a_{d}\right) \in \mathbb{Z}_{p}^{d}$, $\mathbf{b}=\left(b_{1}, \ldots, b_{d}\right) \in \mathbb{Z}_{p}^{d}$.

(1) $\mathcal{P}_{d, p}^{\mathbf{a}, \epsilon}=\mathcal{P}_{d, p}^{\mathbf{b}, \epsilon}$ if and only if there exists $c \in \mathbb{Z}_{p}^{*}$ such that

$$
b_{j} c^{j} \equiv a_{j} \quad(\bmod \quad p) \quad \text { for } \quad j=1, \ldots, d .
$$

(2) If $\mathcal{P}_{d, p}^{\mathbf{a}, \epsilon} \neq \mathcal{P}_{d, p}^{\mathbf{b}, \epsilon}$, then $\mathcal{P}_{d, p}^{\mathbf{a}, \epsilon} \cap \mathcal{P}_{d, p}^{\mathbf{b}, \epsilon}=\{(0, \ldots, 0)\}$.

Proof. (1) We first suppose that there exists $c \in \mathbb{Z}_{p}^{*}$ such that $b_{j} c^{j} \equiv a_{j}(\bmod p)$ for $j \in\{1, \ldots, d\}$. Recall that

$$
\mathcal{P}_{d, p}^{\mathbf{a}, \epsilon}=\left\{\mathbf{x}_{j}^{\mathbf{a}, \epsilon}: j \in \mathbb{Z}_{p}\right\},
$$

where

$$
\mathbf{x}_{j}^{\mathbf{a}, \epsilon}=\left(\left\{\frac{a_{1} j}{p}\right\},\left\{\frac{a_{1}^{\prime} j+a_{2} j^{2}}{p}\right\}, \ldots,\left\{\frac{\sum_{h=1}^{d-1} a_{h}^{\prime} j^{h}+a_{d} j^{d}}{p}\right\}\right) .
$$

For any $j_{0} \in \mathbb{Z}_{p}$, we take $k_{0} \equiv c j_{0} \quad(\bmod \quad p)$. Then

$$
\begin{aligned}
\mathbf{x}_{j_{0}}^{\mathbf{a}, \epsilon} & =\left(\left\{\frac{a_{1} j_{0}}{p}\right\},\left\{\frac{a_{1}^{\prime} j_{0}+a_{2} j_{0}^{2}}{p}\right\}, \ldots,\left\{\frac{\sum_{h=1}^{d-1} a_{h}^{\prime} j_{0}^{h}+a_{d} j_{0}^{d}}{p}\right\}\right) \\
& =\left(\left\{\frac{b_{1} c j_{0}}{p}\right\},\left\{\frac{b_{1}^{\prime} c j_{0}+b_{2} c^{2} j_{0}^{2}}{p}\right\}, \ldots,\left\{\frac{\sum_{h=1}^{d-1} b_{h}^{\prime} c^{h} j_{0}^{h}+b_{d} c^{d} j_{0}^{d}}{p}\right\}\right) \\
& =\left(\left\{\frac{b_{1} k_{0}}{p}\right\},\left\{\frac{b_{1}^{\prime} k_{0}+b_{2} k_{0}^{2}}{p}\right\}, \ldots,\left\{\frac{\sum_{h=1}^{d-1} b_{h}^{\prime} k_{0}^{h}+b_{d} k_{0}^{d}}{p}\right\}\right) \\
& =\mathbf{x}_{k_{0}}^{\mathbf{b}, \epsilon}
\end{aligned}
$$

which implies that

$$
\mathcal{P}_{d, p}^{\mathbf{a}, \epsilon} \subseteq \mathcal{P}_{d, p}^{\mathbf{b}, \epsilon} .
$$

Here we use $b_{j}^{\prime} c^{j} \equiv a_{j}^{\prime}(\bmod p)$ which follows from $b_{j} c^{j} \equiv a_{j}(\bmod p)$.

Since $p$ is a prime number, there exists $c^{-1} \in \mathbb{Z}_{p}^{*}$ so that $c^{-1} c \equiv 1 \quad(\bmod p)$. Then we have $b_{j} \equiv a_{j} c^{-j} \quad(\bmod p), j=1, \ldots, d$. Then, similarly, for any $j_{0} \in \mathbb{Z}_{p}$,

$$
\mathbf{x}_{j_{0}}^{\mathbf{b}, \epsilon}=\mathbf{x}_{c^{-1} j_{0}}^{\mathbf{a}, \epsilon}
$$

which implies that

$$
\mathcal{P}_{d, p}^{\mathbf{b}, \epsilon} \subseteq \mathcal{P}_{d, p}^{\mathbf{a}, \epsilon}
$$


Then we arrive at

$$
\mathcal{P}_{d, p}^{\mathbf{a}, \epsilon}=\mathcal{P}_{d, p}^{\mathbf{b}, \epsilon}
$$

We next suppose that $\mathcal{P}_{d, p}^{\mathbf{a}, \epsilon}=\mathcal{P}_{d, p}^{\mathbf{b}, \epsilon}$. Then there exist $j_{0}, k_{0} \in \mathbb{Z}_{p}^{*}$ so that $\mathbf{x}_{j_{0}}^{\mathbf{a}}=\mathbf{x}_{k_{0}}^{\mathbf{b}}$, i.e.,

$$
\begin{gathered}
a_{1} j_{0} \equiv b_{1} k_{0} \quad(\bmod \quad p), \\
a_{1}^{\prime} j_{0}+a_{2} j_{0}^{2} \equiv b_{1}^{\prime} k_{0}+b_{2} k_{0}^{2} \quad(\bmod \quad p), \\
\vdots \\
\sum_{h=1}^{d-1} a_{h}^{\prime} j_{0}^{h}+a_{d} j_{0}^{d} \equiv \sum_{h=1}^{d-1} b_{h}^{\prime} k_{0}^{h}+b_{d} k_{0}^{d} \quad(\bmod \quad p) .
\end{gathered}
$$

We set $c: \equiv k_{0} j_{0}^{-1} \quad(\bmod p)$, where $j_{0}^{-1} \in \mathbb{Z}_{p}$ so that $j_{0}^{-1} j_{0} \equiv 1 \quad(\bmod p)$. Then (5) implies that $a_{1} \equiv b_{1} c \quad(\bmod p)$. Combining (5) and (6) $)$, we have $a_{2} j_{0}^{2} \equiv b_{2} k_{0}^{2} \quad(\bmod p)$ which implies that $a_{2} \equiv b_{2} c^{2} \quad(\bmod \quad p)$. Similarly, we can obtain that $a_{j} \equiv b_{j} c^{j} \quad(\bmod \quad p)$ for $3 \leq j \leq d$.

(2) We prove it by contradiction. Assume that $\mathcal{P}_{d, p} \cap \mathcal{P}_{d, q} \neq\{(0, \ldots, 0)\}$, and then there exist $j_{0}, k_{0} \in \mathbb{Z}_{p}^{*}$ so that $\mathbf{x}_{j_{0}}^{\mathbf{a}, \epsilon}=\mathbf{x}_{k_{0}}^{\mathbf{b}, \epsilon}$. Similarly with the above proof, we can find a $c: \equiv k_{0} j_{0}^{-1} \quad(\bmod \quad p)$ so that $a_{j} \equiv b_{j} c^{j} \quad(\bmod \quad p)$ for $j=1, \ldots, d$. It leads to $\mathcal{P}_{d, p}^{\mathbf{a}, \epsilon}=\mathcal{P}_{d, p}^{\mathbf{b}, \epsilon}$ by (1) of Theorem 2.4, which is impossible by the assumption in (2).

We next consider the case where $\epsilon^{\prime} \neq \epsilon^{\prime \prime}$.

Theorem 2.5. Suppose that $\epsilon^{\prime}, \epsilon^{\prime \prime} \in\{0,1\}^{d-1}$ with $\epsilon^{\prime} \neq \epsilon^{\prime \prime}$. Set

$$
\mathcal{Z}:=\left\{j: \epsilon_{j}^{\prime} \neq \epsilon_{j}^{\prime \prime} \text { and } a_{j}^{2}+b_{j}^{2} \neq 0,1 \leq j \leq d-1\right\},
$$

and

Then the followings hold.

$$
\ell_{0}:=\min \{j: j \in \mathcal{Z}\}
$$

(1) $\mathcal{P}_{d, p}^{\mathbf{a}, \epsilon^{\prime}}=\mathcal{P}_{d, p}^{\mathbf{b}, \epsilon^{\prime \prime}}$ if and only if there exists a $c \in \mathbb{Z}_{p}^{*}$ so that

$$
\begin{aligned}
& a_{j} \equiv b_{j} c^{j} \quad(\bmod \quad p) \quad \text { for } \quad \epsilon_{j}^{\prime}=\epsilon_{j}^{\prime \prime} \quad \text { or } \quad j=d, \\
& a_{j}=b_{j}=0 \quad \text { for } \quad \epsilon_{j}^{\prime} \neq \epsilon_{j}^{\prime \prime},
\end{aligned}
$$

where $\mathbf{a}, \mathbf{b} \in \mathbb{Z}_{p}^{d} \backslash\{(0, \ldots, 0)\}$ with $a_{1} \neq 0$ being given.

(2) Assume that $\mathcal{P}_{d, p}^{\mathbf{a}, \epsilon^{\prime}} \neq \mathcal{P}_{d, p}^{\mathbf{b}, \epsilon^{\prime \prime}}$ where $\mathbf{a}, \mathbf{b} \in \mathbb{Z}_{p}^{d}$. If $\mathcal{Z}=\emptyset$ then we have

$$
\mathcal{P}_{d, p}^{\mathbf{a}, \epsilon^{\prime}} \cap \mathcal{P}_{d, p}^{\mathbf{b}, \epsilon^{\prime \prime}}=\{(0, \ldots, 0)\} .
$$

If $\mathcal{Z} \neq \emptyset$, then $\left|\mathcal{P}_{d, p}^{\mathbf{a}, \epsilon^{\prime}} \cap \mathcal{P}_{d, p}^{\mathbf{b}, \epsilon^{\prime \prime}}\right| \leq r+1$, where

$$
r=\min \left\{j: a_{j}^{2}+b_{j}^{2} \neq 0\right\} .
$$

(3) Assume that $\mathcal{Z} \neq \emptyset$, and $a_{1} \neq 0$. If $a_{\ell_{0}+1} b_{1}^{\ell_{0}+1} \equiv a_{1}^{\ell_{0}+1} b_{\ell_{0}+1}(\bmod p)$, then $\mathcal{P}_{d, p}^{\mathbf{a}, \epsilon^{\prime}} \cap \mathcal{P}_{d, p}^{\mathbf{b}, \epsilon^{\prime \prime}}=\{(0, \ldots, 0)\}$.

Proof. (1) We assume that (7) holds. Take

$$
\epsilon_{j}=\left\{\begin{array}{cc}
\epsilon_{j}^{\prime}, & \text { if } \epsilon_{j}^{\prime}=\epsilon_{j}^{\prime \prime} \\
0, & \text { if } \epsilon_{j}^{\prime} \neq \epsilon_{j}^{\prime \prime}
\end{array} .\right.
$$

Noting that $a_{j}=b_{j}=0$ provided $\epsilon_{j}^{\prime} \neq \epsilon_{j}^{\prime \prime}$, we have $\mathcal{P}_{d, p}^{\mathbf{a}, \epsilon}=\mathcal{P}_{d, p}^{\mathbf{a}, \epsilon^{\prime}}$ and $\mathcal{P}_{d, p}^{\mathbf{b}, \epsilon}=\mathcal{P}_{d, p}^{\mathbf{b}, \epsilon^{\prime \prime}}$. Theorem 2.4 implies that $\mathcal{P}_{d, p}^{\mathbf{a}, \epsilon}=\mathcal{P}_{d, p}^{\mathbf{b}, \epsilon}$ and hence $\mathcal{P}_{d, p}^{\mathbf{a}, \epsilon^{\prime}}=\mathcal{P}_{d, p}^{\mathbf{b}, \epsilon^{\prime \prime}}$. 
We next assume that $\mathcal{P}_{d, p}^{\mathbf{a}, \epsilon^{\prime}}=\mathcal{P}_{d, p}^{\mathbf{b}, \epsilon^{\prime \prime}}$ which is equivalent to that there exists a permutation $\left\{k_{0}, k_{1}, \ldots, k_{p-1}\right\}$ of $\{0,1, \ldots, p-1\}$ so that

$$
\mathbf{x}_{j}^{\mathbf{a}, \epsilon^{\prime}}=\mathbf{x}_{k_{j}}^{\mathbf{b}, \epsilon^{\prime \prime}}, \quad j=0,1, \ldots, p-1 .
$$

This is equivalent to

$$
\begin{aligned}
a_{1} & \equiv b_{1} k_{1} \quad(\bmod \quad p) \\
2 a_{1} & \equiv b_{1} k_{2} \quad(\bmod \quad p)
\end{aligned}
$$

$$
(p-1) a_{1} \equiv b_{1} k_{p-1} \quad(\bmod \quad p)
$$

and

$$
\begin{aligned}
\sum_{h=1}^{i-1} a_{h}^{\prime}+a_{i} & \equiv \sum_{h=1}^{i-1} b_{h}^{\prime} k_{1}^{h}+b_{i} k_{1}^{i} \quad(\bmod \quad p) \\
\sum_{h=1}^{i-1} 2^{h} a_{h}^{\prime}+a_{i} 2^{i} & \equiv \sum_{h=1}^{i-1} b_{h}^{\prime} k_{2}^{h}+b_{i} k_{2}^{i} \quad(\bmod \quad p) \\
\vdots & \\
\sum_{h=1}^{i-1}(p-1)^{h} a_{h}^{\prime}+a_{i}(p-1)^{i} & \equiv \sum_{h=1}^{i-1} b_{h}^{\prime} k_{p-1}^{h}+b_{i} k_{p-1}^{i} \quad(\bmod \quad p),
\end{aligned}
$$

for $i=2, \ldots, d$. Since $a_{1} \neq 0$, by (9) we have

$$
\begin{aligned}
a_{1} & \equiv b_{1} k_{1} \quad(\bmod p) \\
k_{2} & \equiv 2 k_{1} \quad(\bmod p) \\
& \vdots \\
k_{p-1} & \equiv(p-1) k_{1} \quad(\bmod p) .
\end{aligned}
$$

Set $j_{0}:=\min \left\{i: \epsilon_{i}^{\prime} \neq \epsilon_{i}^{\prime \prime}\right\}$. Using the same argument with the one in Theorem 2.4 we have $a_{i} \equiv b_{i} k_{1}^{i} \quad(\bmod p), i=1, \ldots, j_{0}$. Combining (10) for $i=j_{0}+1$ and (11) we have

$$
\begin{aligned}
& a_{j_{0}}^{\prime}+a_{j_{0}+1} \equiv b_{j_{0}}^{\prime} k_{1}^{j_{0}}+b_{j_{0}+1} k_{1}^{j_{0}+1} \quad(\bmod \quad p) \\
& 2^{j_{0}} a_{j_{0}}^{\prime}+2^{j_{0}+1} a_{j_{0}+1} \equiv b_{j_{0}}^{\prime} k_{2}^{j_{0}}+b_{j_{0}+1} k_{2}^{j_{0}+1}(\bmod p) \text {. }
\end{aligned}
$$

Without loss of generality, we can assume $\epsilon_{j_{0}}^{\prime}=1$ and $\epsilon_{j_{0}}^{\prime \prime}=0$ and then

$$
\begin{aligned}
& a_{j_{0}}+a_{j_{0}+1} \equiv b_{j_{0}+1} k_{1}^{j_{0}+1} \quad(\bmod \quad p) \\
& 2^{j_{0}} a_{j_{0}}+2^{j_{0}+1} a_{j_{0}+1} \equiv b_{j_{0}+1} k_{2}^{j_{0}+1} \quad(\bmod \quad p),
\end{aligned}
$$

which implies $a_{j_{0}}=b_{j_{0}}=0$ since $k_{2} \equiv 2 k_{1} \quad(\bmod \quad p)$ and $a_{j_{0}} \equiv b_{j_{0}} k_{1}^{j_{0}} \quad(\bmod \quad p)$.

(2) We first assume that $\mathcal{Z}=\emptyset$ which implies $a_{j}=b_{j}=0$ provided $\epsilon_{j}^{\prime} \neq \epsilon_{j}^{\prime \prime}$. Take

$$
\epsilon_{j}=\left\{\begin{array}{cc}
\epsilon_{j}^{\prime}, & \text { if } \epsilon_{j}^{\prime}=\epsilon_{j}^{\prime \prime} \\
0, & \text { if } \epsilon_{j}^{\prime} \neq \epsilon_{j}^{\prime \prime}
\end{array} .\right.
$$

Noting that $a_{j}=b_{j}=0$ provided $\epsilon_{j}^{\prime} \neq \epsilon_{j}^{\prime \prime}$, we have $\mathcal{P}_{d, p}^{\mathbf{a}, \epsilon}=\mathcal{P}_{d, p}^{\mathbf{a}, \epsilon^{\prime}}$ and $\mathcal{P}_{d, p}^{\mathbf{b}, \epsilon}=\mathcal{P}_{d, p}^{\mathbf{b}, \epsilon^{\prime \prime}}$. The (2) of Theorem 2.4 implies that $\mathcal{P}_{d, p}^{\mathbf{a}, \epsilon^{\prime}} \cap \mathcal{P}_{d, p}^{\mathbf{b}, \epsilon^{\prime \prime}}=\{(0, \ldots, 0)\}$. We next consider the case where 
$\mathcal{Z} \neq \emptyset$. Suppose that $\mathcal{P}_{d, p}^{\mathbf{a}, \epsilon^{\prime}}$ and $\mathcal{P}_{d, p}^{\mathbf{b}, \epsilon^{\prime \prime}}$ have a common nonzero point. Then, there exist $j, k \in \mathbb{Z}_{p}^{*}$ so that

$$
\begin{aligned}
a_{1} j & \equiv b_{1} k \quad(\bmod \quad p) \\
a_{1}^{\prime} j+a_{2} j^{2} & \equiv b_{1}^{\prime} k+b_{2} k^{2} \quad(\bmod \quad p) \\
& \vdots \\
\sum_{h=1}^{d-1} a_{h}^{\prime} j^{h}+a_{d} j^{d} & \equiv \sum_{h=1}^{d-1} b_{h}^{\prime} k^{h}+b_{d} k^{d} \quad(\bmod \quad p) .
\end{aligned}
$$

Note that $a_{h}=b_{h}=0$ when $h \leq r-1$ and $a_{h}^{\prime}=b_{h}^{\prime}=0, h \leq r-1$. The (12) implies that

$$
\begin{gathered}
a_{h} j^{h} \equiv b_{h} k^{h} \quad(\bmod \quad p), \quad h=r, \ldots, \ell_{0}, \\
a_{\ell}^{\prime} j^{\ell_{0}}+a_{\ell_{0}+1} j^{\ell_{0}+1} \equiv b_{\ell_{0}}^{\prime} k^{\ell_{0}}+b_{\ell_{0}+1} k^{\ell_{0}+1} \quad(\bmod p) .
\end{gathered}
$$

Without loss of generality, we can assume $\epsilon_{\ell_{0}}^{\prime}=1$ and $\epsilon_{\ell_{0}}^{\prime \prime}=0$. By (14), we have

$$
a_{\ell_{0}} j^{\ell_{0}}+a_{\ell_{0}+1} j^{\ell_{0}+1} \equiv b_{\ell_{0}+1} k^{\ell_{0}+1} \quad(\bmod \quad p) .
$$

Taking $h=r$ in (13), we have $a_{r} \equiv b_{r}\left(k j^{-1}\right)^{r} \quad(\bmod \quad p)$ where $j^{-1} \in \mathbb{Z}_{p}$ satisfies $j^{-1} j \equiv$ $1(\bmod p)$. Since $a_{r}^{2}+b_{r}^{2} \neq 0$, we have $a_{r} \neq 0$. Set $x_{0}=k j^{-1}$. Then $x_{0}$ satisfies

$$
\begin{aligned}
a_{r} & \equiv b_{r} x_{0}^{r} \quad(\bmod \quad p) \\
a_{\ell_{0}} j^{-1}+a_{\ell_{0}+1} & \equiv b_{\ell_{0}+1} x_{0}^{\ell_{0}+1} \quad(\bmod \quad p) .
\end{aligned}
$$

Each nonzero point in $\mathcal{P}_{d, p}^{\mathbf{a}, \epsilon^{\prime}} \cap \mathcal{P}_{d, p}^{\mathbf{b}, \epsilon^{\prime \prime}}$ corresponds to a solution to

$$
\begin{aligned}
a_{r} & \equiv b_{r} x^{r} \quad(\bmod \quad p) \\
a_{\ell_{0}} j^{-1}+a_{\ell_{0}+1} & \equiv b_{\ell_{0}+1} x^{\ell_{0}+1} \quad(\bmod p) .
\end{aligned}
$$

Note that $a_{r} \equiv b_{r} x^{r} \quad(\bmod p)$ has at most $r$ solutions. Hence,

$$
\left|\mathcal{P}_{d, p}^{\mathbf{a}, \epsilon^{\prime}} \cap \mathcal{P}_{d, p}^{\mathbf{b}, \epsilon^{\prime \prime}}\right| \leq r+1
$$

(3) We prove it by contradiction. Assume that $\mathcal{P}_{d, p}^{\mathbf{a}, \epsilon^{\prime}} \cap \mathcal{P}_{d, q}^{\mathbf{a}, \epsilon^{\prime \prime}} \neq\{(0, \ldots, 0)\}$, and then there exist $j_{0}, k_{0} \in \mathbb{Z}_{p}^{*}$ so that $\mathbf{x}_{j_{0}}^{\mathbf{a}, \epsilon^{\prime}}=\mathbf{x}_{k_{0}}^{\mathbf{b}, \epsilon^{\prime \prime}}$. Particularly, we have

$$
\begin{gathered}
a_{h} j_{0}^{h} \equiv b_{h} k_{0}^{h} \quad(\bmod p), h=1, \ldots, \ell_{0}, \\
a_{\ell_{0}}^{\prime} j_{0}^{\ell_{0}}+a_{\ell_{0}+1} j_{0}^{\ell_{0}+1} \equiv b_{\ell_{0}}^{\prime} k_{0}^{\ell_{0}}+b_{\ell_{0}+1} k_{0}^{\ell_{0}+1} \quad(\bmod p) .
\end{gathered}
$$

Without loss of generality, we can assume $\epsilon_{\ell_{0}}^{\prime}=1$ and $\epsilon_{\ell_{0}}^{\prime \prime}=0$. By (17), we have

$$
a_{\ell_{0}} j_{0}^{\ell_{0}}+a_{\ell_{0}+1} j_{0}^{\ell_{0}+1} \equiv b_{\ell_{0}+1} k_{0}^{\ell_{0}+1} \quad(\bmod \quad p) .
$$

By (16)) with $h=1$, we have

$$
\begin{aligned}
a_{\ell_{0}+1} j_{0}^{\ell_{0}+1}-b_{\ell_{0}+1} k_{0}^{\ell_{0}+1} & \equiv a_{\ell_{0}+1}\left(b_{1} k_{0} a_{1}^{-1}\right)^{\ell_{0}+1}-b_{\ell_{0}+1} k_{0}^{\ell_{0}+1} \\
& \equiv k_{0}^{\ell_{0}+1}\left(a_{\ell_{0}+1} b_{1}^{\ell_{0}+1} a_{1}^{-\ell_{0}-1}-b_{\ell_{0}+1}\right) \\
& \equiv 0(\bmod p),
\end{aligned}
$$

according to $a_{\ell_{0}+1} b_{1}^{\ell_{0}+1} \equiv a_{1}^{\ell_{0}+1} b_{\ell_{0}+1} \quad(\bmod \quad p)$. By (18) $)$, we have $a_{\ell_{0}} j_{0}^{\ell_{0}} \equiv 0 \quad(\bmod p)$, which implies that $a_{\ell_{0}} \equiv 0 \quad(\bmod p)$ or $j_{0} \equiv 0 \quad(\bmod p)$. This is impossible by the assumption. 
In the following, we choose the appropriate vectors $\mathbf{a}, \mathbf{b}$ so that $\left|\mathcal{L}_{p, q}\right|=q+p-1$. We now state the inequalities for exponential sums over $\mathcal{L}_{p, q}$, which is the main result of this subsection.

Theorem 2.6. Suppose $p$ and $q$ are odd prime numbers and set $m=p+q$. Recall that

$$
\mathcal{L}_{p, q}=\left\{\begin{array}{cc}
\mathcal{P}_{d, p} \cup \mathcal{P}_{d, q}, & p \neq q \\
\mathcal{P}_{d, p}^{\mathbf{a}, \epsilon^{\prime}} \cup \mathcal{P}_{d, p}^{\mathbf{b}, \epsilon^{\prime \prime}}, & p=q .
\end{array}\right.
$$

We assume that $\left|\mathcal{L}_{p, q}\right|=p+q-1$. Then, for any $\mathbf{k} \in[-p+1, p-1]^{d} \cap[-q+1, q-1]^{d} \cap \mathbb{Z}^{d}$ and $\mathbf{k} \neq 0$, we have

$$
\left|\sum_{\mathbf{x} \in \mathcal{L}_{p, q}} \exp (2 \pi \mathbf{i k} \cdot \mathbf{x})\right| \leq(d-1) \sqrt{2 m}+1 .
$$

Proof. We first consider the case where $p=q$. We have

$$
\mathcal{L}_{p, q}=\mathcal{P}_{d, p}^{\mathbf{a}, \epsilon^{\prime}} \cup \mathcal{P}_{d, p}^{\mathbf{b}, \epsilon^{\prime \prime}}
$$

Recall that

$$
\mathcal{P}_{d, p}^{\mathbf{a}, \epsilon^{\prime}} \cap \mathcal{P}_{d, p}^{\mathbf{b}, \epsilon^{\prime \prime}}=\{(0, \ldots, 0)\}
$$

Then

$$
\begin{aligned}
\left|\sum_{\mathbf{x} \in \mathcal{L}_{p, q}} \exp (2 \pi \mathbf{i} \mathbf{k} \cdot \mathbf{x})\right| & \leq\left|\sum_{\mathbf{x} \in \mathcal{P}_{d, p}^{\mathbf{a}, \epsilon^{\prime}}} \exp (2 \pi \mathbf{i} \mathbf{k} \cdot \mathbf{x})\right|+\left|\sum_{\mathbf{x} \in \mathcal{P}_{d, p}^{\mathbf{b}, \epsilon^{\prime \prime}}} \exp (2 \pi \mathbf{i k} \cdot \mathbf{x})\right|+1 \\
& \leq(d-1) \sqrt{p}+(d-1) \sqrt{p}+1 \\
& =(d-1) \sqrt{2 m}+1
\end{aligned}
$$

Here, in the last inequality, we use Theorem 2.2 We next consider the case where $p \neq q$. When $p \neq q, \mathcal{L}_{p, q}=\mathcal{P}_{d, p} \cup \mathcal{P}_{d, q}$. Then we have

$$
\begin{aligned}
\left|\sum_{\mathbf{x} \in \mathcal{L}_{p, q}} \exp (2 \pi \mathbf{i k} \cdot \mathbf{x})\right| & \leq\left|\sum_{\mathbf{x} \in \mathcal{P}_{d, p}} \exp (2 \pi \mathbf{i k} \cdot \mathbf{x})\right|+\left|\sum_{\mathbf{x} \in \mathcal{P}_{d, q}} \exp (2 \pi \mathbf{i k} \cdot \mathbf{x})\right|+1 \\
& \leq(d-1) \sqrt{p}+(d-1) \sqrt{q}+1 \\
& \leq(d-1) \sqrt{2 m}+1 .
\end{aligned}
$$

2.3. The exponential sums over $\mathcal{Q}_{p^{2}, d}^{\mathbf{a}, \epsilon}$ and $\mathcal{R}_{p^{2}, d}^{\mathbf{a}, \epsilon}$. Suppose that $\mathbf{a} \in \mathbb{Z}_{p}^{d}$ and $\epsilon \in$ $\{0,1\}^{d-1}$. We set

$$
\begin{gathered}
\mathcal{Q}_{p^{2}, d}^{\mathbf{a}, \epsilon}:=\left\{\mathbf{z}_{j}^{\mathbf{a}, \epsilon}: j=0, \ldots, p^{2}-1\right\}, \\
\mathbf{z}_{j}^{\mathbf{a}, \epsilon}=\left(\left\{\frac{a_{1} j}{p^{2}}\right\},\left\{\frac{a_{1}^{\prime} j+a_{2} j^{2}}{p^{2}}\right\}, \ldots,\left\{\frac{\sum_{h=1}^{d-1} a_{h}^{\prime} j^{h}+a_{d} j^{d}}{p^{2}}\right\}\right) \in[0,1)^{d}
\end{gathered}
$$

and

$$
\begin{gathered}
\mathcal{R}_{p^{2}, d}^{\mathbf{a}, \epsilon}:=\left\{\mathbf{z}_{j, k}^{\mathbf{a}, \epsilon}: j, k=0, \ldots, p-1\right\}, \\
\mathbf{z}_{j, k}^{\mathbf{a}, \epsilon}=\left(\left\{\frac{a_{1} k}{p}\right\},\left\{\frac{\left(a_{1}^{\prime}+a_{2} j\right) k}{p}\right\}, \ldots,\left\{\frac{\left(\sum_{h=1}^{d-1} a_{h}^{\prime} j^{h-1}+a_{d} j^{d-1}\right) k}{p}\right\}\right) \in[0,1)^{d} .
\end{gathered}
$$

The $\mathcal{Q}_{p^{2}, d}^{\mathbf{a}, \epsilon}$ and $\mathcal{R}_{p^{2}, d}^{\mathbf{a}, \epsilon}$ can be considered as the generalization of the $p$-sets given in (3). Based on the Lemma 5 and Lemma 6 in [2], we can obtain the following inequalities for exponential sums over $\mathcal{Q}_{p^{2}, d}^{\mathbf{a}, \epsilon}$ and $\mathcal{R}_{p^{2}, d}^{\mathbf{a}, \epsilon}$. 
Theorem 2.7. Suppose that $\mathbf{a} \in \mathbb{Z}_{p}^{d}$ and $\epsilon \in\{0,1\}^{d-1}$. Then, for any $\mathbf{k}=\left(k_{1}, \ldots, k_{d}\right) \in$ $[-p+1, p-1]^{d} \cap \mathbb{Z}^{d}$ and $\mathbf{k} \neq 0$, we have

$$
\left|\sum_{\mathbf{x} \in \mathcal{Q}_{p^{2}, d}^{\mathbf{a}, \epsilon}} \exp (2 \pi \mathbf{i k} \cdot \mathbf{x})\right| \leq(d-1) p .
$$

Proof. Set

$$
g(j):=\sum_{\ell=1}^{d} c_{\ell} j^{\ell}
$$

where $c_{\ell}=k_{\ell} a_{\ell}+k_{\ell+1} a_{\ell}^{\prime}+\cdots+k_{d} a_{\ell}^{\prime}$. We set $j_{0}:=\max \left\{\ell: k_{\ell} \neq 0\right\}$. Then $c_{j_{0}}=k_{j_{0}} a_{j_{0}}$ and we have $p \nmid c_{j_{0}}$. According to Lemma 5 in [2], we have

$$
\left|\sum_{\mathbf{x} \in \mathcal{Q}_{p^{2}, d}^{\mathbf{a}, \epsilon}} \exp (2 \pi \mathbf{i k} \cdot \mathbf{x})\right|=\left|\sum_{j=0}^{p^{2}-1} \exp \left(2 \pi \mathbf{i} \frac{g(j)}{p^{2}}\right)\right| \leq(d-1) p .
$$

Theorem 2.8. Suppose that $\mathbf{a} \in[1, p-1]^{d} \cap \mathbb{Z}^{d}$. Then, for any $\mathbf{k} \in[-p+1, p-1]^{d} \cap \mathbb{Z}^{d}$ and $\mathbf{k} \neq 0$, we have

$$
\left|\sum_{\mathbf{x} \in \mathcal{R}_{p^{2}, d}^{\mathbf{a}, \epsilon}} \exp (2 \pi \mathbf{i k} \cdot \mathbf{x})\right| \leq(d-1) p
$$

Proof. Set

$$
g(j):=\sum_{\ell=0}^{d-1} c_{\ell} j^{\ell}
$$

and $c_{\ell}:=k_{\ell+1} a_{\ell+1}+k_{\ell+2} a_{\ell+1}^{\prime}+\cdots+k_{d} a_{\ell+1}^{\prime}$. We set $j_{0}:=\max \left\{\ell: k_{\ell} \neq 0\right\}$. Then $c_{j_{0}-1}=$ $k_{j_{0}} a_{j_{0}}$ and we have $p \nmid c_{j_{0}-1}$. Using Lemma 6 in [2], we have

$$
\left|\sum_{\mathbf{x} \in \mathcal{R}_{p^{2}, \epsilon}^{\mathbf{a}, \epsilon}} \exp (2 \pi \mathbf{i k} \cdot \mathbf{x})\right|=\left|\sum_{j=0}^{p-1} \sum_{k=0}^{p-1} \exp \left(2 \pi \mathbf{i} k \frac{g(j)}{p}\right)\right| \leq(d-1) p .
$$

\section{The Applications of $\mathcal{P}_{d, p}^{\mathbf{a}, \epsilon}$ AND $\mathcal{L}_{p, q}$}

Based on the exponential sum formula in Section 2, the new point sets are useful in numerical integration [8, 1], in UQ [13] and in the recovery of sparse trigonometric polynomials [12. We just state the results for the recovery of sparse trigonometric polynomials in detail.

We start with some notations which go back to [12. Set

$$
\Pi_{s}^{d}:=\left\{f: f(\mathbf{x})=\sum_{\mathbf{k} \in[-s, s]^{d} \cap \mathbb{Z}^{d}} c_{\mathbf{k}} e^{2 \pi \mathbf{i} \mathbf{k} \cdot \mathbf{x}}, \quad c_{\mathbf{k}} \in \mathbb{C}, \quad \mathbf{x} \in[0,1]^{d}\right\} .
$$

Note that $\Pi_{s}^{d}$ is a linear space with the dimension $D:=(2 s+1)^{d}$. For

$$
f(\mathbf{x})=\sum_{\mathbf{k} \in[-s, s]^{d} \cap \mathbb{Z}^{d}} c_{\mathbf{k}} e^{2 \pi \mathbf{i k} \cdot \mathbf{x}} \in \Pi_{s}^{d},
$$

we set $\mathbf{T}:=\left\{\mathbf{k}: c_{\mathbf{k}} \neq 0\right\}$ which is the support of the sequence of coefficients $c_{\mathbf{k}}$, and set

$$
\Pi_{s}^{d}(M):=\bigcup_{\mathbf{T} \subset[-s, s]^{d} \cap \mathbb{Z}^{d},|\mathbf{T}| \leq M} \Pi_{\mathbf{T}},
$$


where $\Pi_{\mathbf{T}}$ denotes the space of all trigonometric polynomials whose coefficients are supported on $\mathbf{T}$. When $M \ll D$, we call the trigonometric polynomials in $\Pi_{s}^{d}(M)$ as $M$-sparse trigonometric polynomials.

The recovery of sparse trigonometric polynomials is an active topic recently. The main aim of this research topic is to design a sampling set $X=\left\{\mathbf{z}_{j}\right\}_{j=1}^{N}$ so that one can recover $f \in \Pi_{s}^{d}(M)$ from $f\left(\mathbf{z}_{j}\right), \mathbf{z}_{j} \in X[12,9$, 6]. We state the problem as follows. Assume the sampling set is $X=\left\{\mathbf{x}_{j} \in[0,1)^{d}, j=1, \ldots, N\right\}$. Then our aim is to solve the following programming:

$$
\text { find } f \in \Pi_{s}^{d}(M) \quad \text { subject to } \quad f\left(\mathbf{x}_{j}\right)=y_{j}, \quad j=1, \ldots, N .
$$

Denote by $\mathbf{F}_{X}$ the $N \times D$ sampling matrix with entries

$$
\left(\mathbf{F}_{X}\right)_{j, \mathbf{k}}=\exp \left(2 \pi \mathbf{i k} \cdot \mathbf{x}_{j}\right), \quad j=1, \ldots, N, \quad \mathbf{k} \in[-s, s]^{d} \cap \mathbb{Z}^{d} .
$$

Let $\mathbf{a}_{\mathbf{k}}=\left(\exp \left(2 \pi \mathbf{i k} \cdot \mathbf{x}_{j}\right)\right)_{j=1}^{N}$ denote a column of $\mathbf{F}_{X}$ with $\mathbf{k} \in[-s, s]^{d} \cap \mathbb{Z}^{d}$. A simple observation is that $\left\|\mathbf{a}_{\mathbf{k}}\right\|_{2}=\sqrt{N}$. Set

$$
\mu:=\mu_{X}:=\frac{1}{N} \max _{\mathbf{m}, \mathbf{k} \in[-s, s]^{d} \cap \mathbb{Z}^{d}, \mathbf{m} \neq \mathbf{k}}\left|\left\langle\mathbf{a}_{\mathbf{m}}, \mathbf{a}_{\mathbf{k}}\right\rangle\right|,
$$

which is called the mutual incoherence of the matrix $\mathbf{F}_{X} / \sqrt{N}$. Theorem 2.5 in [6] shows that if $\mu<1 /(2 M-1)$ then the Orthogonal Matching Pursuit Algorithm (OMP) and the Basis Pursuit Algorithm (BP) can recover any $M$-sparse trigonometric polynomials in $\Pi_{s}^{d}(M)$. Therefore, our aim is to choose the sampling set $X$ so that $\mu$ is small and hence OMP and BP can recover $M$-sparse trigonometric polynomials. Based on Theorem 2.2 and Theorem 2.6 respectively, the following results give upper bounds of $\mu$ with taking $X=\mathcal{P}_{d, p}^{\mathbf{a}, \epsilon}$, and $X=\mathcal{L}_{p, q}$, respectively.

Lemma 3.1. (1) Suppose that $X=\mathcal{P}_{d, p}^{\mathbf{a}, \epsilon}$ where $\mathbf{a} \in[1, p-1]^{d} \cap \mathbb{Z}^{d}$ and $p \geq 2 s+1$ is a prime number. Then

$$
\mu_{X} \leq(d-1) / \sqrt{p}
$$

(2) Suppose that $p, q \geq 2 s+1$ are prime numbers and $\mathbf{a}, \mathbf{b} \in[1, p-1]^{d} \cap \mathbb{Z}^{d}$. Recall that

$$
\mathcal{L}_{p, q}=\left\{\begin{array}{cc}
\mathcal{P}_{d, p} \cup \mathcal{P}_{d, q}, & p \neq q \\
\mathcal{P}_{d, p}^{\mathbf{a}, \epsilon^{\prime}} \cup \mathcal{P}_{d, p}^{\mathbf{b}, \epsilon^{\prime \prime}}, & p=q .
\end{array}\right.
$$

Set $X=\mathcal{L}_{p, q}$ and $m=p+q$. Then

$$
\mu_{X} \leq \frac{(d-1) \sqrt{2 m}+1}{m-1} .
$$

As said before, if $\mu<1 /(2 M-1)$ then OMP (and also BP) can recover every $M$-sparse trigonometric polynomials. Then we have the following corollary:

Theorem 3.2. (1) Suppose that $p>\max \left\{2 s+1,(d-1)^{2}(2 M-1)^{2}+1\right\}$ is a prime number and $\mathbf{a} \in[1, p-1]^{d} \cap \mathbb{Z}^{d}$. Then $O M P$ (and also $B P$ ) recovers every $M$-sparse trigonometric polynomial $f \in \Pi_{s}^{d}(M)$ exactly from the deterministic sampling $\mathcal{P}_{d, p}^{\mathbf{a}, \epsilon}$.

(2) Under the condition in (2) of Lemma 3.1. Suppose that

$$
m=p+q>\left(\left(\frac{1}{\sqrt{2}}+\frac{1}{2}\right)(2 M-1)(d-1)+\sqrt{M}\right)^{2} .
$$

Then OMP (and also BP) recovers every $M$-sparse trigonometric polynomial $f \in$ $\Pi_{s}^{d}(M)$ exactly from the deterministic sampling set $\mathcal{L}_{p, q}$. 
Proof. We first consider (1). Note that $p \geq(d-1)^{2}(2 M-1)^{2}+1$ implies that $(d-1) / \sqrt{p}<$ $1 /(2 M-1)$. According to (1) in Lemma 3.1, if $(d-1) / \sqrt{p}<1 /(2 M-1)$ then $\mu<1 /(2 M-1)$ and hence the conclusion follows. Similarly, we can prove $(2)$.

\section{REFERENCES}

[1] J. Dick, Numerical integration of Hölder continuous, absolutely convergent Fourier-, Fourier cosine-, and Walsh series, J. Approx. Theory, 183, 14-30, 2014.

[2] J. Dick and F. Pillichshammer, The weighted star discrepancy of Korobov's $p$-sets, Proceedings of the American Mathematical Society, 143(12), 5043-5057, 2015.

[3] Michael Drmota, Robert F. Tichy, Sequences, discrepancies and applicationa, Lecture Notes in Mathematics, vol. 1651, Springer-Verlag, Berlin, 1997.

[4] L. K. Hua and Y. Wang, Applications of number theory to numerical analysis. Springer-Verlag, BerlinNew York, 1981. Translated from the chinese edition, Science Press, Beijing, 1978.

[5] N. M. Korobov, Number-theoretic methods in approximate analysis. Gosudarstv. Izdat. Fiz.-Mat. Lit., Moscow, 1963.

[6] S. Kunis, H. Rauhut, Random sampling of sparse trigonometric polynomials II- Orthogonal matching pursuit versus basis pursuit, Foundations of Computational Mathematics, 8(6), 1615-3375, 2008.

[7] Gunther Leobacher, Friedrich Pillichshammer, Introduction to quasi-Monte Carlo integration and applications, Compact Textbook in Mathematics, Birkhäuser/Springer, Cham, 2014.

[8] H. Niederreiter, Random Number Generation and Quasi-Monte Carlo Methods, CBMS-NSF Series in Applied Mathematics, vol. 63, SIAM, Philadelphia, 1992.

[9] H. Rauhut. Random sampling of sparse trigonometric polynomials, Appl. Comput. Harmon. Anal., 22, 16-42, 2007.

[10] Ian H. Sloan, Henryk Woźniakowski, When are quasi-Monte Carlo algorithms efficient for highdimensional integrals?, J. Complexity, 14, 14(1), 1-33, 1998.

[11] A. Weil, On some exponential sums, PNAS, USA, 34, 204-207, 1948.

[12] Z. Xu, Deterministic sampling of sparse trigonometric polynomials, Journal of Complexity, 27(2), 133$140,2011$.

[13] T. Zhou, A. Narayan and Z. Xu, Multivariate discrete least-squares approximations with a new type of collocation grid, SIAM J. Sci. Comput., 36(5), A2401-A2422, 2014.

School of Sciences, Tianjin Polytechnic University, Tianjin 300160, China

E-mail address: zhouheng7598@sina.com.cn

LSEC, Institute of Computational Mathematics, Academy of Mathematics and System Sciences, Chinese Academy of Sciences, Beijing 100190, China

E-mail address: xuzq@lsec.cc.ac.cn 